Published in final edited form as:

Contemp Clin Trials. 2016 November ; 51: 88-95. doi:10.1016/j.cct.2016.10.009.

\title{
Weight management for adolescents with intellectual and developmental disabilities: Rationale and design for an 18 month randomized trial
}

\author{
JE Donnellya, ${ }^{\star}$, LT Ptomeya ${ }^{\mathrm{a}}$, JR Goetz ${ }^{\mathrm{c}}$, DK Sullivan ${ }^{\mathrm{c}}$, CA Gibson ${ }^{\mathrm{d}}$, JL Greene $^{\mathrm{b}}$, RH Lee $^{\mathrm{e}}$, MS \\ Mayo $^{f}, J^{J}$ Honas $^{a}$, and RA Washburn ${ }^{a}$ \\ a Cardiovascular Research Institute, Department of Internal Medicine, The University of Kansas \\ Medical Center, 3901 Rainbow Boulevard, Kansas City, KS, 66160 USA. \\ b Department of Health, Sport, and Exercise Sciences, University of Kansas, Lawrence, KS \\ 66045, USA \\ c Department of Dietetics and Nutrition, The University of Kansas Medical Center, 3901 Rainbow \\ Boulevard, Kansas City, KS, 66160 USA. \\ d Department of Internal Medicine, The University of Kansas Medical Center, 3901 Rainbow \\ Boulevard, Kansas City, KS, 66160 USA. \\ eDepartment of Health Policy and Management, The University of Kansas Medical Center, 3901 \\ Rainbow Boulevard, Kansas City, KS, 66160 USA. \\ f Department of Biostatistics, The University of Kansas Medical Center, 3901 Rainbow Boulevard, \\ Kansas City, KS, 66160 USA.
}

\section{Abstract}

\begin{abstract}
Adolescents with intellectual and developmental disabilities (IDD) are an underserved group in need of weight management. However, information regarding effective weight management for this group is limited, and is based primarily on results from small, non-powered, non-randomized trials that were not conducted in accordance with current weight management guidelines.

Additionally, the comparative effectiveness of emerging dietary approaches, such as portioncontrolled meals (PCMs) or program delivery strategies such as video chat using tablet computers have not been evaluated. Therefore, we will conduct an 18 month trial to compare weight loss (6
\end{abstract}

\footnotetext{
*Corresponding author: Joseph E. Donnelly, Robinson Center, Rm. 100, The University of Kansas-Lawrence, 1301 Sunnyside Avenue, Lawrence, KS 66045, Phone: 785-864-0797, Fax: 785-864-2009, jdonnelly@ku.edu.

Lauren T. Ptomey (lptomey@ kumc.edu)

J. Leon Greene (jlg@ku.edu)

Richard A. Washburn (rwashburn@ku.edu)

Debra K. Sullivan (dsulliva@kumc.edu)

Cheryl Gibson (cgibson@kumc.edu)

Jeannine Goetz (jgoetz@kumc.edu)

Jeff Honas (jhonas@ku.edu)

Robert Lee (rlee2@kumc.edu)

Matthew S Mayo (mmayo@kumc.edu)

Publisher's Disclaimer: This is a PDF file of an unedited manuscript that has been accepted for publication. As a service to our customers we are providing this early version of the manuscript. The manuscript will undergo copyediting, typesetting, and review of the resulting proof before it is published in its final citable form. Please note that during the production process errors may be discovered which could affect the content, and all legal disclaimers that apply to the journal pertain.
} 
mos.) and maintenance (7-18 mos.) in 123 overweight/obese adolescents with mild to moderate IDD, and a parent, randomized to a weight management intervention delivered remotely using FaceTime $^{\mathrm{TM}}$ on an iPad using either a conventional meal plan diet (RD/CD) or a Stop Light diet enhanced with PCMs (RD/eSLD), or conventional diet delivered during face-to-face home visits (FTF/CD). This design will provide an adequately powered comparison of both diet (CD vs. eSLD) and delivery strategy (FTF vs. RD). Exploratory analyses will examine the influence of behavioral session attendance, compliance with recommendations for diet (energy intake), physical activity (min/day), self-monitoring of diet and physical activity, medications, and parental variables including diet quality, physical activity, baseline weight, weight change, and beliefs and attitudes regarding diet and physical activity on both weight loss and maintenance. We will also complete a cost and contingent valuation analysis to compare costs between RD and FTF delivery.

\section{Keywords}

intellectual and developmental disability; diet; weight management; physical activity; adolescents; technology; Stop Light Diet

\section{INTRODUCTION}

Intellectual and developmental disabilities (IDD) are characterized by significant limitations in both intellectual functioning (IQ $<75$ ) and in adaptive behavior including conceptual skills (e.g. language and literacy, self-direction), social skills (e.g. interpersonal skills, ability to follow rules) and practical skills (e.g. personal care, use of money/phone) which originate before the age of 18 [1]. The prevalence of overweight and obesity (BMI $\geq$ the $85^{\text {th }} \%$ ile) in adolescents with IDD (age 11-18 yrs.) in the United States is $~ 50 \%$ [2] [3] which is considerably higher than the prevalence of overweight and obesity in general adolescent population $(\sim 35 \%)$ [4]. The premature morbidity and mortality in adulthood associated with overweight/obesity in typically developing children and adolescents likely applies to adolescents with IDD [5] although data specific to adolescents with IDD are unavailable.

Adolescents with IDD are an underserved group in need of weight management; however, the limitations associated with IDD, and the altered dynamics of interactions with parents and siblings preclude the extrapolation of weight management strategies shown to be effective in typically developing adolescents to this group. Unfortunately, the quality and quantity of data on which to base recommendations for effective weight management specifically for adolescents with IDD is limited. The most recent systematic review evaluating weight loss interventions in youth/adolescents with IDD identified only 9 trials published between 1985 and 2013 [2]. The majority of trials were non-randomized (8/9, $89 \%$ ), with interventions recommending increased physical activity with no dietary component ( $7 / 9, \sim 78 \%$ ), conducted over short durations ( $<6 \mathrm{mos}$.), in small samples with inadequate statistical power $(\mathrm{n}<30)$. These interventions were not conducted in accordance with current recommendations for the treatment of child and adolescent overweight and obesity, which comprise 1) a planned negative energy balance achieved through structured diet and physical activity, 2) a structured behavior modification program that includes selfmonitoring of both diet and physical activity, and 3) weekly (at least 8-12 weeks in duration) 
followed by monthly individual or group counseling to change and maintain diet and physical activity behaviors [6]. Not unexpectedly, weight loss reported in these trials was minimal ( $\leq 3 \%$ ). A trial published subsequent to the Maiano et al [2] review compared weight loss in overweight and obese adolescents and young adults with Down syndrome (13-26 yrs.) randomized to a 6-month nutrition and physical activity education intervention $(\mathrm{n}=10)$ or a nutrition and physical activity education plus parent-supported behavioral intervention $(\mathrm{n}=11)$ with a 6-month no contact follow-up [7]. Both groups included a reduced energy diet and increased physical activity; however, only the parent-supported group included a structured behavioral modification program as suggested by current adolescent weight loss guidelines [6]. Results indicated weight change at both 6 and 12 months was significantly greater in the group with parental support $(6$ mos. $=-3.4 \% ; 12$ mos. $=-2.4 \%)$ compared with the non-parental support groups $(6$ mos. $=+0.6 \% ; 12$ mos. $=$ $+2.2 \%)$. However, the magnitude of weight loss at 12 months in the parental support group was not clinically meaningful ( $\leq 3 \%)[8]$.

The limited available literature and the generally small magnitude of reported weight loss suggests that additional innovative weight management strategies for adolescents with IDD need to be developed and evaluated. Our group has conducted preliminary work to evaluate the feasibility and effectiveness of an enhanced version of the Stop Light Diet (eSLD) delivered remotely to adolescents with IDD and a parent, in their homes, via video chat on an iPad (FaceTime ${ }^{\mathrm{TM}}$, Apple, Inc., Cupertino, CA) [9]. The Stop Light Diet, originally developed for use in children[10], classifies foods by energy content into 3 categories associated with a traffic light: green (low energy - consume freely), yellow (moderate energy - consume in moderation) and red (high energy - consume sparingly). We enhanced the Stop Light Diet by recommending daily consumption of $2 \sim 200$ kcal portion-controlled entrées, $2 \sim 100 \mathrm{kcal}$ shakes, 5 servings of fruits/vegetables, and ad-libitum non-caloric beverages. Participants desiring additional food selected food items categorized as low energy (i.e., green) using the SLD system. The eSLD is easy to understand and simplifies meal planning, and food shopping/meal preparation, thus making it potentially effective for use with individuals with IDD. We recently compared 2-month weight loss in 20 overweight/obese adolescents with IDD and his/her parent who were randomized to either an eSLD $(n=11)$ or a conventional meal plan diet $(\mathrm{CD}, n=11)$ and increased physical activity [9]. The participant/parent dyads were asked to attend weekly 30-45 minute behavioral sessions conducted by a trained health educator delivered on an iPad using FaceTime ${ }^{\mathrm{TM}}$ (Apple Inc. Cupertino, CA). Participants, with assistance from a parent, were asked to self-monitor daily dietary intake using a web-based application (Lose It!, Fitnow Inc., Boston, MA) using the iPad. Physical activity was monitored using a pedometer and by recording daily steps on a paper $\log (\mathrm{n}=13)$ or using a wireless activity tracker(Fitbit Ultra, Fitbit Inc., San Francisco, CA) which automatically uploaded physical activity data to the iPad $(n=7)$. Results indicated participants attended $84 \%$ of scheduled weekly behavioral sessions; $80 \%$ using FaceTime ${ }^{\mathrm{TM}}$, and $4 \%$ by phone when away from home where internet access was unavailable. Dietary intake using the Lose It! app on the iPad was recorded on $83 \%$ of study days. Self-monitoring of physical activity in the 7 participants who used the Fitbit was completed on $72 \%$ of study days compared with $60 \%$ of study days in the 13 participants who recorded pedometer steps using paper logs. Clinically meaningful weight loss was 
observed in both the eSLD and CD groups $(-4.6 \%$ vs $-3.1 \%, p=0.12)$ at 2 months. These encouraging preliminary results led to the development of the current randomized trial designed to compare the effectiveness of two diets (eSLD vs. CD), and two delivery strategies (face-to-face (FTF)) vs. remote delivery (RD) using FaceTime ${ }^{\mathrm{TM}}$ for both weight loss and maintenance in adolescents with IDD.

\section{METHODS AND MATERIALS}

\section{Overview of study design (Table 1)}

One hundred twenty-three overweight/obese adolescents (ages 13-21) with mild to moderate IDD along with a parent will be randomized to one of three groups for an 18-month trial (6 mos. weight loss, 12 mos. maintenance): RD/CD, RD/eSLD or FTF/CD. The adolescent/ parent dyad will attend 30-45 minute behavioral sessions with a health educator twice per month during weight loss (0-6 mos.) and the first 6 months of weight maintenance (7-12 mos.), and once per month during the last 6 months of weight maintenance (13-18 mos.). All groups will be prescribed a reduced energy diet (0-6 mos.) and a diet with energy intake to support weight maintenance (7-18 mos.) plus a program on increased moderate intensity physical activity (0-18 mos.). The remote intervention (RD) will be delivered on an iPad using FaceTime ${ }^{\mathrm{TM}}$, an application for real-time video conferencing. Self-monitoring in the RD group will be completed using web-based apps for diet (Lose it!), physical activity (Fitbit), and weight (wireless scales). The FTF intervention will be delivered during homevisits. Self-monitoring will be completed by participants with the help of a parent through pencil and paper records for diet, pedometers for physical activity and weight assessed by research staff during home visits. The primary aim is to compare weight loss from baseline to 6 months among the 3 randomized groups. Secondary aims will compare weight maintenance between month 7 to month 18 among the 3 randomized groups and compare costs of delivery between FTF and RD using a cost and contingent valuation analysis. We will also explore the influence of the following participant and parent variables on weight loss (0-6 mos.) and maintenance (7-18 mos.): Participant variables include behavioral session attendance, compliance with recommendations for diet (energy intake), physical activity (min/day), self-monitoring of diet and physical activity, and medications. Parent variables collected to assess the potential influences of parental changes in energy intake, physical activity and weight on adolescent weight change will include: diet quality, physical activity, baseline weight, weight change across 18 months, and beliefs and attitudes regarding diet and physical activity.

\section{Participant eligibility}

Adolescents will be required to designate one parent to serve as the primary family contact. To better assist their adolescent with intervention compliance, the parent will be asked to attend all behavioral sessions to familiarize themselves with both the diet and physical activity recommendations and the behavioral strategies incorporated in the intervention. The parent will be asked to provide support and encouragement, and provide assistance, if necessary, in following the prescribed diet (e.g., meal planning and preparation), planning and promoting physical activity, and self-monitoring of diet and physical activity. The intervention is designed to target the adolescent; however, we recognize that some parents 
who need to lose weight may choose to diet and engage in physical activity with their adolescents. This will be neither encouraged nor discouraged. Exploratory analysis will assess the potential influences of parental changes in energy intake, physical activity and weight on adolescent weight change. To enhance the generalizability of our results, adolescents who use medications for common chronic diseases (e.g., depression, type 2 diabetes, elevated blood pressure and lipids) or other medications that may compromise weight loss or induce weight gain will not be excluded. Randomization should ensure that medication use is equally distributed across the 3 study groups. Specific participant eligibility criteria are presented in Table 2 .

\section{Recruitment/randomization}

Participants (at least $50 \%$ female) will be recruited through local community programs serving adolescents with IDD, and with print and web advertisements in the target area. All recruitment materials will include contact information for research staff (toll-free phone, email). Questions from interested parents will be addressed and initial eligibility screening will be completed via phone or email. Home visits will be scheduled with interested parents and potential participants to explain the project, answer questions, obtain consent, and determine eligibility. Choice is important for individuals with IDD; thus, adolescent assent will be required for enrollment in addition to parental consent. A separate consent for enrollment will be obtained from the participating parent. Adolescents found to be ineligible will be referred to other appropriate weight management options. Randomization, stratified by BMI\%ile (<95\%ile, $>95 \%$ ile) to ensure balanced allocations, will be completed after obtaining written signed informed consent (parent) and assent (adolescent) and written physician clearance. Treatment allocation sequences will be generated by the project statistician using computer software with equal allocation to the RD/CD, RD/eSLD, and FTF/CD groups. Allocation will be concealed in envelopes and delivered to the study coordinator.

\section{Orientation}

Health educators will conduct 2 home visits ( 90 min. each) with each adolescent and his/her parent prior to initiating the intervention. These sessions will include detailed descriptions of the dietary (eSLD/CD) and physical activity components of intervention, and the respective delivery and self-monitoring formats. Participants in all groups will be provided with an iPad mini. Access to non-study related materials, e.g. web browsing, app store etc., will be blocked on all iPads until completion of the study. iPads for the RD groups will be pre-loaded with the Lose It!, Fitbit, and wireless scale apps (Withings Inc., Cambridge, MA). The RD groups will receive a tutorial on the use of the iPad and will be oriented to FaceTime ${ }^{\mathrm{TM}}$, Lose it!, Fitbit, and wireless scales apps/devices used for selfmonitoring diet, PA, and weight. Time will be allotted for practice and questions. Tutorials describing trouble shooting for common technical problems (e.g., internet connectivity, data entry using Fitbit, Lose It!) will be loaded on the iPad. Technical issues can also be resolved during behavioral sessions, or by contacting research staff by phone or email. Reminders regarding upcoming behavioral sessions or prompts for participants in the RD groups who are non-compliant with the study protocol will be sent via the iPad. The FTF group will also be oriented to the iPad, which will be used to assist with assessment of dietary intake in both 
intervention groups as described below (Assessments). However, no diet or physical activity applications will be loaded on the iPad. Participants in the FTF group will be provided with a pedometer (Omron HJ-320, Lake Forest, IL) to self-monitor physical activity and will be shown how to self-monitor diet and PA using paper records specifically designed for use with individuals with IDD.

\section{Intervention components: Diet}

Enhanced Stop Light Diet (eSLD)—The original SLD, developed by Epstein for use in children [10], is an effective weight loss strategy for children [11] as indicated by a Grade 1 ranking (strong, consistent, supporting evidence) from the Academy of Nutrition and Dietetics Evidence Analysis Library [12]. The SLD categorizes foods by energy content: green (low-consume freely), yellow (moderate-consume in moderation), and red (high consume sparingly). The SLD is easy to understand and implement and thus is appropriate for adolescents with IDD with assistance from their parents. Lists and/or pictures of foods that are color-coded based on the Stop Light diet that can be used in meal planning shopping and snack selection, will be provided to all participants. As in our pilot trial, we will "enhance" the Stop Light Diet (eSLD) and encourage the consumption of high volume, low energy portion controlled meals (PCMs, entrées /shakes; HRM Weight Management Services Corp, Boston, MA) and fruits and vegetables. The use of PCMs simplifies meal planning, food shopping and meal preparation, which may be important for adolescents with IDD and their parents. The Academy of Nutrition and Dietetics Evidence Analysis Library indicates Grade 1 support for the use of PCMs in individuals who have difficulty with selfselection of food and portion control [13]. PCMs from HMR are shelf-stable, do not require refrigerated storage, and can be prepared with a microwave (entrées) or blender (shakes). Participants will be encouraged to consume a minimum of 2 entrées (200-270 kcal each), 2 shakes ( $100 \mathrm{kcal}$ each) and 5 one-cup servings of fruits and vegetables each day. Noncaloric beverages will be allowed ad libitum. Participants wanting food in addition to the prescribed PCMs and fruits/vegetables will be encouraged to use the SLD color-coded chart to select additional servings of lower energy foods. PCMs will be provided as part of the eSLD only during weight loss (0-6 mos.), and will be shipped to the participant's homes every other week. During weight maintenance (7-18 mos.), participants will be encouraged (not required) to continue using PCMs. A list of recommended low calorie/fat PCMs that are available for purchase at most grocery stores will be provided to participants and parents at month 7 .

Conventional meal plan diet (CD)—Participants in the 2 groups prescribed a CD (RD/CD and FTF/CD) will be asked to consume a nutritionally balanced, reduced energy, high volume, lower fat (fat=20-30\% energy) diet as recommended by the Academy of Nutrition and Dietetics [14] and the USDA's MyPlate approach [15]. Participants will be provided with examples of meal plans consisting of suggested servings of grains, proteins, fruits and vegetables, dairy, and fats based on their energy needs and will be counseled on appropriate portion sizes. Consistent with the eSLD, the consumption of 5 one-cup servings of fruits and vegetables per day will be encouraged. To assist in offsetting any additional cost associated with dietary compliance, participants in the $\mathrm{CD}$ groups will receive $\$ 2.00 /$ day in the form of a university issued debit card that will be recharged monthly. 


\section{Intervention components: Energy Intake}

Energy intake for weight loss (0-6 mos.) will be reduced 500-700 kcal/d below total daily energy expenditure estimated using the Dietary Reference Intake (DRI) total energy equation for overweight boys/girls age 3-18 yrs. [16] in both diet groups (CD/eSLD). We are aware that this equation was not developed for use in adolescents with IDD. Consistent with our pilot trial, we will use this estimate as a reasonable starting point and make adjustments as necessary depending on observed weight change. We will ensure that both weight loss diets will be nutritionally adequate for growth and development (i.e., energy intake $>1,200$ $\mathrm{kcal} / \mathrm{d},>20 \%$ of total energy intake from fat, RDA vitamins, minerals)[17]. Energy intake for weight maintenance (7-18 mos.) will also be estimated using the DRI equation [16] with consideration for growth and development and adjusted as required based on observed changes in weight. Participants experiencing weight gain will be counseled to improve compliance to their prescribed level of energy intake and physical activity during their regularly scheduled meetings with health educators. Participants who do not meet their weight loss goals may voluntarily attempt additional weight loss during weight maintenance and this will be neither encouraged nor discouraged.

\section{Intervention components: Physical activity}

All participants will be asked to reach a target of $60 \mathrm{~min}$./day of moderate intensity physical activity (3-6 METs) at least 5 days/wk. (total $300 \mathrm{~min} / \mathrm{wk}$.) as recommended by the US Department of Health and Human Services [18]. The initial recommendation will be 45 min./wk. (or current activity level if higher) during week one, increasing by $15 \mathrm{~min} . / \mathrm{wk}$. until reaching the $300 \mathrm{~min} . /$ wk. goal. A variety of activities including walking, swimming, biking, active video games (e.g., Dance Dance Revolution ${ }^{\mathrm{TM}}$, Wii Sports ${ }^{\mathrm{TM}}$ ), and recreational sports outside of school will be promoted using guidelines and suggestions from the "Train at Home" program of the Special Olympics. An adapted physical education specialist will assist participants and parents in modifying activities to accommodate any special needs.

\section{Intervention components: Behavioral session schedule/content}

Participants and parents in all groups will attend $\sim 30-45$ min. sessions with a health educator twice per month during weight loss ( $0-6$ mos.) and the first 6 months of weight maintenance (7-12 mos.), and once per month during the last 6 months of weight maintenance (13-18 mos.). Sessions will include behavioral strategies to improve weight loss/maintenance and include topics such as social support, self-monitoring, planning, environmental control, selfefficacy, etc. Identical session content will be loaded on the iPads of the RD groups or provided as a booklet for the FTF group. In addition to the lesson, health educators will review diet and physical activity data, current weight status, answer questions, problemsolve, and provide support at each session.

\section{Intervention components: Self-Monitoring}

All intervention groups will be asked to monitor food intake and physical activity daily, and body weight bi-weekly across the 18-month intervention. Participants in the RD/CD and RD/eSLD groups will record all food and beverages consumed (meals/snacks) using the Lose it! app on the iPad by entering the food name and selecting the portion size, or by 
scanning the bar code of the food item using the iPad. As a food is entered, a bar graph comparing actual with recommended energy intake is displayed, providing participants with immediate feedback regarding compliance with their energy intake goal. Diet data on the Lose it! app are automatically and securely transferred via the web to the Ascend for Wellness Professionals web site (Fitnow Inc. Boston, MA). These data are accessible to health educators to inform participant counseling during behavioral sessions. Physical activity in the RD/CD and RD/eSLD groups will be tracked using a Fitbit Charge HR wireless activity tracker (size $35.5 \times 28 \mathrm{~mm}$ ) worn on the wrist. The Fitbit records time spent in light, moderate or vigorous activity, using step counts and heart rate. These data are automatically transferred to the Lose it! app via Bluetooth when the device is near the iPad, thus eliminating the need for manual data recording. A graphic display on the Lose it! app provides immediate feedback on participants accumulated physical activity relative to their goal. Fitbit data are automatically transferred to cloud storage (Fitabase-Small Steps Labs LLC, San Diego, CA) where they are available to health educators for use in participant counseling. Reminders will be programmed into the Lose It! app to prompt participants who fail to provide diet information or to sync the Fitbit to transfer physical activity data. To provide feedback regarding weight loss/gain, participants in the RD groups will self-weigh bi-weekly (0-18 mos.) during a FaceTime ${ }^{\mathrm{TM}}$ session using a calibrated wireless digital scale (Model: WS-30, Withings Inc. Cambridge, MA). Weight data automatically syncs with the Lose it! app and updates a visual display of weight change. Participants/parents dyads in the FTF group will be asked to record the number of servings of fruits, vegetables, grains, protein foods, and dairy consumed using a paper form containing pictorial representations of each food category. These forms also provide space for recording the duration of physical activity and pedometer steps. These records will be collected by health educators during behavioral session home visits and will be used as motivation, and to inform participant counseling for the FTF group. Body weight to provide participant feedback regarding weight loss or gain will be assessed on a calibrated digital scale (Model \#PS6600, Belfour, Saukville, WI) during one of the monthly behavioral sessions. Participants will be provided with a personal weight loss "histogram" with color coded bands indicating a change of approximately one BMI \%ile. Self-monitoring data (diet/physical activity) from all intervention groups will be transferred/entered in a database for inclusion in our exploratory analyses.

\section{Health Educator training, intervention fidelity, blinding}

Health educators will have a minimum of a bachelors degree in nutrition, exercise science, behavioral psychology or related field and 1-2 years experience in weight management with individuals with IDD. All health educators will train for a minimum of 3 months by shadowing a health educator experienced in working with adolescents with IDD. All health educators will participate in weekly 2 -hour staff meetings to discuss specific issues related to intervention delivery. Health educators will be randomly assigned to participants in each of the 3 intervention groups to diminish health educator bias. All health educator/participant sessions (FaceTime ${ }^{\mathrm{TM}} / \mathrm{FTF}$ ) will be recorded. Intervention fidelity will be assessed by comparing recordings with a check list of content to be delivered. Health educators who deliver $<80 \%$ of intended content will be counseled prior to their next participant meeting. The nature of our intervention precludes blinding of the health educators; however, 
investigators, data analysts and research assistants who collect outcome data will be blinded to condition.

\section{Incentives}

All participants will be allowed to keep their iPad on completing the trial at which time staff will unlock access to all iPad features that had been blocked during the trial. As an additional incentive, participants in all groups who complete self-monitoring for diet and physical activity on 5 of 7 days/wk. will be allowed to choose an iPad app from a list of age appropriate apps. Research staff will remotely upload the apps to the participant's iPad. All participants and parents will receive gift cards ( $\$ 15$ participants, $\$ 10$ parents) for completing each of the 4 outcome assessments. Participants remaining in the trial at 6,12 and 18 months will be eligible to select a gift, such as a water bottle, resistance band, t-shirt, yoga mat etc. These gifts will be distributed by health educators during a separate home visit in the RD groups, and during a regularly scheduled home visit in the FTF group. The separate home visit is designed to maintain rapport between health educators, participants and parents.

\section{ASSESSMENTS}

Most outcomes will be assessed during a single home visit at baseline, 6, 12, and 18 months; however, some assessments will occur across the 18-month intervention (Table 3) Physical measures (weight, height, waist circumference) will be obtained from adolescents and their designated parent during a home visit, between 7-10 a.m., following a minimum 12-hour fast. All assessments will be conducted by trained staff blinded to the condition. Staff will receive refresher training and complete reliability assessments for all physical measures 2-3 times each year. We estimate measures obtained during the home visit will require approximately 60 minutes to complete.

\section{Outcomes: Primary/Secondary Aims}

Weight/height/waist circumference-Weight will be measured, in duplicate, to the nearest $0.1 \mathrm{~kg}$ using a calibrated digital scale (Model \#PS6600, Belfour, Saukville, WI) with participants wearing shorts and a t-shirt. Standing height will be measured in duplicate with a portable stadiometer (Model \#IP0955, Invicta Plastics Limited, Leicester, UK). BMI \%ile will be calculated using the Centers for Disease Control's BMI \%ile calculator for children and teens (http://apps.nccd.cdc.gov). Waist circumference will be measured using the procedures described by Lohman et al [19]. Three measurements will be obtained with the outcome recorded as the average of the closest 2 measures.

Cost Measures-A potential advantage to remote delivery may be lower costs for both participants and providers, thus we will compare costs for the RD and FTF groups.

\section{Outcomes-Exploratory aims}

Compliance with behavioral sessions-The percentage of possible behavioral sessions (0-6 mos. and 7-18 mos.) will be calculated from attendance data maintained by the health educator. 
Compliance with diet recommendations-Energy and macronutrient intake will be assessed using photo-assisted food records over 3 consecutive days ( 2 week days, 1 weekend day) starting the weekend prior to the scheduled outcome assessments. Our group [29, $30]$ and others [31, 32] have demonstrated the utility of photo-assisted food records for dietary assessment in adults with IDD. Participants with assistance of a parent, if necessary, will take before and after photographs of all foods and beverages consumed using the iPad camera, which automatically date and time stamps photos for easy identification. Hard copy records will be completed to account for details not discernible from photos (e.g. skim or whole milk). During outcome visits, staff will review photos and food records and clarify ambiguities. If no photo is available for a particular meal, food record data will be used. Recalls will be used in cases where food record data are unavailable. To facilitate recording, instructions describing how to complete the food record and take food photos, and calendar prompts to remind participants to comply with the protocol, will be loaded/programmed on the iPad. We will calculate the average energy expenditure $(\mathrm{kcal} / \mathrm{d})$, macronutrient intake $(\%$ carbs, fat, protein), and servings of fruits and vegetables using the Nutrition Data System for Research (Version 2015, Minneapolis, MN).

Compliance with physical activity recommendations-Physical activity will be assessed over 7 consecutive days using an ActiGraph Model GT3X+ portable accelerometer (ActiGraph LLC, Pensacola, FL). ActiGraphs will be distributed during scheduled home outcome assessment visits and returned to research staff via pre-paid mail. Adolescents will be asked to wear the accelerometer on a belt over the non-dominant hip during all waking hours. A minimum of four 10-hour days at each assessment time point will be considered a valid observation [33]. Parents will be asked to assist their adolescent in achieving compliance with the monitoring protocol. The outcome will be the average minutes per day spent in moderate-to-vigorous physical activity over the 7-day period, calculated using the age-specific intensity cut points suggested by Trost et al [34].

Self-monitoring of diet and physical activity-The percentage days for which participants provide data (0-6 mos. and 7-18 mos.) for diet and physical activity will used to assess compliance with the self-monitoring protocol.

Medications-Medication information (name/frequency and amount will be obtained at each outcome assessment. Medications will be classified (yes/no) as to their association with weight gain/loss [35].

\section{Parent outcomes}

Weight/height-Weight will be measured, in duplicate, to the nearest $0.1 \mathrm{~kg}$ using a calibrated digital scale (Model \#PS6600, Belfour, Saukville, WI) with parents wearing shorts and a t-shirt. Standing height will be measured in duplicate with a portable stadiometer (Model \#IP0955, Invicta Plastics Limited, Leicester, UK).

Diet-The National Cancer Institute's All-Day Fruit and Vegetable screener [36] will be used to assess changes in parent fruit and vegetable consumption over the over the course of the intervention. 
Physical activity-Parent physical activity will be assessed using the International Physical Activity Questionnaire short form which has been shown to provide valid assessments of moderate/vigorous physical activity over the past 7 days in adults [37, 38].

Parent's beliefs/attitudes-Parent's beliefs/attitudes regarding diet and physical activity will be assessed using an adapted version of the Healthy Buddies Parent Nutrition and Physical Activity Survey which has been previously used with parents of adolescents with IDD [39]. Survey items assess perceptions about the child's weight, beliefs about the importance and frequency of family meals, and the frequency of fast food consumption. Additional items assess parents' beliefs, attitudes, and behaviors about their family's eating habits, and physical activity.

\section{Parent/participant interview}

Semi-structured telephone interviews will be conducted in a random sample of 36 parent/ participant dyads (12/group, $30 \%$ of total sample) at 6 and 18 months to solicit feedback on a range of topics. For example, parents will be asked how they learned about the study, what motivated them to enroll, their perceptions of the intervention, their perceptions of the selfefficacy of the participant to follow the diet, set goals, and self-monitor, as well as any challenges or barriers encountered during the study. Parents and participants will be asked to describe both positive and negative aspects of the intervention, including issues related to convenience, satisfaction with the respective diets, ease of participation in both counseling and self-monitoring (pencil/paper vs. iPad), level of satisfaction with intervention components and whether the services provided in the intervention met their expectations. In addition to overall satisfaction, we will ask parents to identify attributes of the intervention important to their family (e.g., timeliness, flexibility of treatment scheduling, etc.). To encourage unreserved and descriptive accounts of their experiences, research staff conducting the interviews will not be involved in intervention delivery or outcome assessments; thus, they will not be acquainted with either parents or participants. We will also conduct semi-structured phone interviews with parents of participants who drop from the study to explore reasons for drop out and to solicit suggestions for improving the intervention. All interviews will be recorded, transcribed verbatim, and entered into a qualitative data analysis software program (Atlas.ti 7, Berlin, Germany). Content analysis of the transcribed interviews will be completed using the method suggested by Miles and Huberman [40] and further described by Ryan and Bernard [41] to explore trends and identify common themes across interviews using an inductive method. Coding disagreements will be brought to the research team for review and discussed until $100 \%$ agreement is reached.

\section{Statistical power/sample size}

We considered a 2 by 2 factorial (4 group) design (i.e. including a FTF/eSLD group); however, the sample size required for that design was in excess of 240 total participants (i.e. $>80$ per group) and was considered impractical from a cost, logistical, and recruitment perspective. Thus, we chose a 3 group design which allows a comparison of effect of both diet (CD vs. eSLD) and delivery strategy (FTF vs. RD) in a cost efficient manner. Based on the data from our preliminary trial [9] we expect significantly greater weight loss for $\mathrm{RD} / \mathrm{CD}$ 
(- $4.0 \mathrm{~kg})$ compared to FTF/CD $(-1.5 \mathrm{~kg})$ and significantly greater weight loss for $\mathrm{RD} /$ eSLD $(-6.5 \mathrm{~kg})$ compared to RD/CD $(-4.0 \mathrm{~kg})$ at 6 months with a common standard deviation of $3.6 \mathrm{~kg}$. With these weight loss assumptions, 41 participants in each of the 3 intervention groups (123 total) would provide over $99 \%$ power for an overall ANOVA comparing the three groups for a global difference, however we are only interested in the two pair-wise comparisons described above and chose our sample size to ensure sufficient power for those pair-wise comparisons. Thus, this design results in $80 \%$ power to detect the hypothesized differences with a type 1 error rate of 0.025 for each of the 2 pair-wise comparisons of interest from the overall ANOVA. This Bonferroni adjustment is very conservative adjustment to the type one error rate to ensure our overall type I error rate is less than or equal to 5\%. Based on our preliminary trail in adolescents with IDD [9] we estimate that $85 \%$ of participants in each group will complete the 6-month weight loss phase of the intervention, and we will utilize multiple imputation to impute weight change for those missing 6 month weights for our primary analysis.

\section{Analysis-Primary/Secondary aims}

Weight-Two separate two sample t-tests will be used to compare weight loss (0-6 mos.) between $\mathrm{RD} / \mathrm{CD}$ and RD/eSLD group (i.e. effect of diet) and RD/CD and FTF/CD group (i.e., effect of delivery strategy). The primary comparison will be completed using intent-totreat principles with a secondary completer's only analysis. We will examine the impact of baseline characteristics (age, sex, race/ethnicity, weight, chronic disease) on weight change using linear regression controlling for treatment. We will also examine the main effects of each of these variables, as well as the potential interaction effect with treatment. Missing data for analyses of our primary aim (weight loss 0-6 mos.) will be imputed using multiple imputations. We will determine if the proportion of participants lost to follow-up differs by treatment. If so, we will determine if there are differences in demographic characteristics (sex, age, baseline weight) between completers and those lost to follow-up. If missing data are related to treatment and/or demographic characteristics, we will use model based multiple imputation; otherwise, we will use traditional multiple imputation $(\mathrm{k}=5)$. Two separate t-tests will be used to compare weight changes from 7-18 months as described for our primary aim, but this analysis will only include those subject who have measurements at both time points. We will also use mixed linear models to compare weight between treatment groups from 7-18 mos. controlling for treatment and weight at 6 mos. These assessments will be conducted only in participants who provide weight at 6 months, which represents baseline weight for weight maintenance. We anticipate loss to follow-up will increase over time; however, we expect this missing data to be missing at random. Under this assumption, mixed linear models will provide unbiased treatment comparisons. We will assume an autoregressive correlation structure of the dependent variable (weight) over time, and determine if there are any time-by-treatment interactions. Statistical procedures will be performed using SAS version 9.4 or higher.

Cost-The cost perspective for all analyses will be societal. We will collect data on both program (health educator time, supplies, iPads, food costs) and adolescent/parent costs (time), prospectively. The gold standard for measuring program cost is a time study, based on a validated flowchart [21, 22]. We will validate flowcharts for all study arms and use time 
studies to measure health educator time and program records to measure cost of supplies. Participants' time represents a component of cost, as time devoted to an intervention cannot be used for work or leisure [23]. These costs, which may be half of the total costs [24, 25] will be gathered via survey[26]. Quantifying time costs in adolescents is problematic as there is no agreement as to the value of the competing uses for their time (e.g., education or paid work) [27]. Thus, we will collect time data for adolescents in this study and then conduct sensitivity analyses using alternative measures of the value of time ranging from $\$ 0.00$ to the local median retail wage. We will value the time spent by parents in assisting their adolescent with the intervention at the local median hourly wage. Weight management programs have effects in addition to weight loss (e.g., increased vitality or enhanced attractiveness), thus the value of a program to clients may be imperfectly measured by weight loss. Contingent valuation, in which participants express preferences for programs, will be used to examine the perceived worth of the RD and FTF formats at 6 and 18 months[28]. If the preference questions have acceptable psychometric properties, conditional logistic regressions, with adjustments for clustering by health educator, will be used to analyze how differences in client attributes, and anticipated costs/gains affect preferences for delivery formats.

\section{Analysis-Exploratory aims}

We will examine energy balance variables (energy intake, PA), process variables (selfmonitoring, meeting attendance), parental diet and PA, parental baseline weight and weight change, and psychosocial variables (parent's beliefs and attitudes regarding nutrition and PA) to assess their influence on weight loss at 6 mos. and on weight maintenance (7-18 mos.) mos 7-18. To determine if treatment impacts these variables, we will compare changes in each variable during both weight loss (0-6 mos.6) and weight maintenance (7-18 mos.) 18) across the 3 intervention groups. We will use linear regression techniques to examine the impact of these variables, as well as baseline demographic characteristics (sex, age, race/ ethnicity, BMI\%ile),to identify the best subsets of variables that explain weight loss at 6 mos. and weight maintenance (7-18 mos.) while controlling for treatment. Note: We will examine main effects of each of these variables as well as the potential interaction effect with treatment.

\section{DISCUSSION}

Adolescents with IDD have high levels of obesity compared with typically developing adolescents; however, they are underserved in terms of weight management. The limited number of weight management trials that have included adolescents with IDD have generally used non-randomized designs, and have been conducted over short time frames, in small non-powered samples, and did not prescribe an energy reduced diet [2]. With the exception of our pilot trial [9], we are unware of trials that have evaluated the use of an innovative dietary strategy, which combines the simplicity of the Stop Light Diet and the convenience of PCMs, or a strategy that uses commercially available devices and applications for the delivery and monitoring of a weight management intervention in adolescents with IDD, as we will undertake. We selected the eSLD for this trial for 3 reasons. First, the eSLD simplifies meal planning, food shopping, and meal preparation, 
which represent significant barriers to weight management for adolescents with IDD and their parents, compared with a CD. Second, the eSLD has consistently shown clinically meaningful weight loss in the range of $4 \%$ to $7 \%$ in adults [42] and adolescents with IDD [9] and adults with physical disabilities [43]. Third, the eSLD requires minimal training, which enhances the probability of implementation and dissemination by providers of health-related services for adolescents with IDD.

Computer technology (desktop/tablets etc.) is widely available, and frequently used in the daily lives of both typically developing adolescents and adolescents with IDD [44]. Computers have been successfully used in individuals with IDD across a variety of tasks including education to improve academic [45], vocational [46] and living skills [47]. However, computer technology has been generally underutilized in health promotion programs such as weight management [46]. Remote delivery and self-monitoring of both diet and physical activity using tablet computers, as will be empoyed in this trial, reduces the burden and potentially the cost of participation in weight management which may both improve weight loss outcomes and make weight management accessible to more adolescents with IDD.

This trial will compare weight loss ( $0-6$ mos. $)$ and weight maintenance between two diets (eSLD vs. CD) and two delivery/self-monitoring strategies (RD vs. FTF). All 3 trial arms will provide a reduced energy diet and increased physical activity. Thus, all participants will receive an intervention with the potential for weight loss/maintenance and its associated health benefits. We chose this approach rather than including a classic non-intervention control group for two reasons. First, we expect that overweight and obese adolescents with IDD randomized to a non-intervention control group would not lose weight, and would continue to be at increased risk for chronic diseases, as there are no data in the literature to suggest otherwise. Second, it could be considered unethical to randomize overweight and obese adolescents with IDD, who represent an under-served and vulneralble group clearly in need of effective strategies for weight management, to a non-intervenion control group. We expect clinically significant and greater weight loss and less weight regain in the RD/eSLD compared with the RD/CD and FTF/CD groups. However, we also expect clinically meaningful weight loss in the $\mathrm{RD} / \mathrm{CD}$ and $\mathrm{FTF} / \mathrm{CD}$ groups. If our $\mathrm{RD}$ approach is successful, clinics and agencies providing weight management services to adolescents wih IDD would have an accessible, scalable, and potentially cost-effective alternative for weight management.

Design and methodologic strengths of this trial include: (1) a 3 group design with adequate statistical power for comparisons of diets (CD vs. eSLD) and delivery approaches (RD vs. FTF) for both weight loss and maintenance; (2) the evaluation of commercially available computer technology for both intervention delivery (iPad-FaceTime ${ }^{\mathrm{TM}}$ ) and self-monitoring of diet (Lose it!) and physical activity (Fitbit); (3) A cost analysis and contingent valuation analysis to compare FTF with RD; (4) the use of photo assisted (iPad) diet records to improve the quality of dietary compliance data; (5) delivery of all 3 intervention arms to cohorts of participants by the same health educator to minimize the potential for health educator bias; (6) assessments completed by trained staff blinded to the intervention arm; (7) evaluation of staff inter-rater reliability for all physical assessments (2-3 times/yr.); and , (8) 
structured interviews to obtain potentially useful information for improving and/or implementing the intervention.

In summary, this trial will compare the effectiveness of 2 diets and 2 delivery strategies for weight management in adolescents with IDD. Results from this trial will provide important information regarding potentially effective components and strategies to improve weight management for adolescents with IDD.

\section{Acknowledgments}

Funding: National Institutes of Child Health and Development (R01 HD079643) We acknowledge HRM Weight Management Services Corp, Boston, MA for providing portion-controlled meals.

\section{Abbreviations}

eSLD enhanced Stop Light Diet

IDD Intellectual and developmental disabilities

PCMs Portion controlled meals

RD Remote Delivery

CD Conventional Diet

\section{REFERENCES}

1. American Association on Intellectual and Developmental Disabilities. Definition of Intellectual Disabilities. Washington, D.C.: 2012.

2. Maïano C, Normand CL, Aimé A, Bégarie J. Lifestyle interventions targeting changes in body weight and composition among youth with an intellectual disability: A systematic review. Research in developmental disabilities. 2014; 35:1914-26. [PubMed: 24830882]

3. Foley JT, Lloyd M, Vogl D, Temple VA. Obesity trends of 8-18 year old Special Olympians: 20052010. Research in developmental disabilities. 2014; 35:705-10. [PubMed: 24397890]

4. Ogden CL, Carroll MD, Kit BK, Flegal KM. Prevalence of childhood and adult obesity in the United States, 2011-2012. Jama. 2014; 311:806-14. [PubMed: 24570244]

5. Reilly JJ, Kelly J. Long-term impact of overweight and obesity in childhood and adolescence on morbidity and premature mortality in adulthood: systematic review. International journal of obesity. 2011; 35:891-8. [PubMed: 20975725]

6. Spear BA, Barlow SE, Ervin C, Ludwig DS, Saelens BE, Schetzina KE, et al. Recommendations for treatment of child and adolescent overweight and obesity. Pediatrics. 2007; 120(Suppl 4):S254-88. [PubMed: 18055654]

7. Curtin C, Bandini LG, Must A, Gleason J, Lividini K, Phillips S, et al. Parent Support Improves Weight Loss in Adolescents and Young Adults with Down Syndrome. The Journal of Pediatrics. 2013; 163:1402-8. e1. [PubMed: 23968742]

8. Jensen MD, Ryan DH, Apovian CM, Ard JD, Comuzzie AG, Donato KA, et al. 2013 AHA/ACC/TOS guideline for the management of overweight and obesity in adults: a report of the American College of Cardiology/American Heart Association Task Force on Practice Guidelines and The Obesity Society. Journal of the American College of Cardiology. 2014:63. [PubMed: 24998130]

9. Ptomey LT, Sullivan DK, Lee J, Goetz JR, Gibson C, Donnelly JE. The use of technology for delivering a weight loss program for adolescents with intellectual and developmental disabilities. Journal of the Academy of Nutrition and Dietetics. 2015; 115:112-8. [PubMed: 25441960] 
10. Epstein, L., Squires, S. The Stoplight Diet for Children: An Eight-Week Program for Parents and Children. Little Brown \& Co; Boston: 1988.

11. Epstein LH, Valoski A, Wing RR, McCurley J. Ten-year outcomes of behavioral family-based treatment for childhood obesity. Health Psychology. 1994; 13:373. [PubMed: 7805631]

12. Academy of Nutrition and Dietetics. What is the evidence to support using the Traffic Light Diet to limiting calorie and food intake in children?. Association AD., editor. 2005.

13. Academy of Nutrition and Dietetics. How effective (in terms of client adherence and weight loss and maintenance) are meal replacements (liquid meals, meal bars, frozen prepackaged meals)?. 2011.

14. Raynor HA, Champagne CM. Position of the Academy of Nutrition and Dietetics: Interventions for the Treatment of Overweight and Obesity in Adults. Journal of the Academy of Nutrition and Dietetics. 2016; 116:129-47. [PubMed: 26718656]

15. U.S. Department of Agriculture. Choose MyPlate. Washington DC. 2013

16. Institute of Medicine. Dietary Reference Intakes for Energy, Carbohydrate, Fiber, Fat, Fatty Acids, Cholesterol, Protein, and Amino Acids (Macronutrients). The National Academies Press; Washington DC: 2002.

17. Trumbo P, Schlicker S, Yates AA, Poos M. Dietary reference intakes for energy, carbohydrate, fiber, fat, fatty acids, cholesterol, protein and amino acids. Journal of the American Dietetic Association. 2002; 102:1621. [PubMed: 12449285]

18. United States Department of Health and Human Services. Physical activity guidelines advisory committee report to the Secretary of Health and Human Services. 2008. http:http:// www.health.gov/PAGuidelines/committeereport.aspx

19. Lohman, TG., Roche, AF., Martorell, R. Anthropometric standardization reference manual. Human Kinetics Books; Champaign,Ill: 1988.

20. Drummond MF, Sculpher MJ, Torrance GW. Methods for the economic evaluation of health care programs: Oxford university press. 2005

21. Sloan, FA. Valuing health care: Costs, benefits, and effectiveness of pharmaceuticals and other medical technologies. Cambridge University Press; 1996.

22. Lee RH, Bott MJ, Forbes S, Redford L, Swagerty DL, Taunton RL. Process-based costing. J Nurs Care Qual. 2003; 18:259-66. [PubMed: 14556582]

23. Russell LB, Gold MR, Siegel JE, Daniels N, Weinstein MC. The role of cost-effectiveness analysis in health and medicine. Panel on Cost-Effectiveness in Health and Medicine. Jama. 1996; 276:1172-7. [PubMed: 8827972]

24. Frew E, Wolstenholme JL, Atkin W, Whynes DK. Estimating time and travel costs incurred in clinic based screening: flexible sigmoidoscopy screening for colorectal cancer. Journal of medical screening. 1999; 6:119-23. [PubMed: 10572841]

25. Yabroff KR, Warren JL, Knopf K, Davis WW, Brown ML. Estimating patient time costs associated with colorectal cancer care. Med Care. 2005; 43:640-8. [PubMed: 15970778]

26. Reuben DB, Wong RC, Walsh KE, Hays RD. Feasibility and accuracy of a postcard diary system for tracking healthcare utilization of community-dwelling older persons. J Am Geriatr Soc. 1995; 43:550-2. [PubMed: 7730540]

27. Wight VR, Price J, Bianchi SM, Hunt BR. The time use of teenagers. Social Science Research. 2009; 38:792-809.

28. Narbro K, Sjostrom L. Willingness to pay for obesity treatment. International journal of technology assessment in health care. 2000; 16:50-9. [PubMed: 10815353]

29. Ptomey LT, Herrmann SD, Lee J, Sullivan DK, Rondon MF, Donnelly JE. Photo-Assisted Recall Increases Estimates of Energy and Macronutrient Intake in Adults with Intellectual and Developmental Disabilities. Journal of the Academy of Nutrition and Dietetics. 2013; 113:1704-9. [PubMed: 24095784]

30. Ptomey LT, Willis EA, Goetz JR, Lee J, Sullivan DK, Donnelly JE. Digital photography improves estimates of dietary intake in adolescents with intellectual and developmental disabilities. Disability and health journal. 2015; 8:146-50. [PubMed: 25281035] 
31. Humphries K, Traci MA, Seekins T. Food on FIlm: Pilot Test of an Innovative Method for Recording Food Intake of Adults with Intellectual Disabilities Living in the Community. Journal of Applied Research in Intellectual Disabilities. 2008; 21:126-73.

32. Elinder L, Brunosson A, Bergström H, Hagströmer M, Patterson E. Validation of personal digital photography to assess dietary quality among people with intellectual disabilities. Journal of Intellectual Disability Research. 2012; 56:221-6. [PubMed: 21801265]

33. Troiano RP, Berrigan D, Dodd KW, Masse LC, Tilert T, McDowell M. Physical activity in the United States measured by accelerometer. Medicine and science in sports and exercise. 2008; 40:181-8. [PubMed: 18091006]

34. Trost SG, Pate RR, Sallis JF, Freedson PS, Taylor WC, Dowda M, et al. Age and gender differences in objectively measured physical activity in youth. Medicine and science in sports and exercise. 2002; 34:350-5. [PubMed: 11828247]

35. Domecq JP, Prutsky G, Leppin A, Sonbol MB, Altayar O, Undavalli C, et al. Clinical review: Drugs commonly associated with weight change: a systematic review and meta-analysis. J Clin Endocrinol Metab. 2015; 100:363-70. [PubMed: 25590213]

36. Thompson FE, Subar AF, Smith AF, Midthune D, Radimer KL, Kahle LL, et al. Fruit and vegetable assessment: performance of 2 new short instruments and a food frequency questionnaire. Journal of the American Dietetic Association. 2002; 102:1764-72. [PubMed: 12487538]

37. Kim Y, Park I, Kang M. Convergent validity of the international physical activity questionnaire (IPAQ): meta-analysis. Public health nutrition. 2013; 16:440-52. [PubMed: 22874087]

38. Craig CL, Marshall AL, Sjostrom M, Bauman AE, Booth ML, Ainsworth BE, et al. International physical activity questionnaire: 12-country reliability and validity. Medicine and science in sports and exercise. 2003; 35:1381-95. [PubMed: 12900694]

39. George VA, Shacter SD, Johnson PM. BMI and attitudes and beliefs about physical activity and nutrition of parents of adolescents with intellectual disabilities. Journal of intellectual disability research : JIDR. 2011; 55:1054-63. [PubMed: 21726317]

40. Miles, MB., Huberman, AM. Qualitative data analysis. 2nd. Edition. Thousand Oaks: 1994.

41. Ryan GW, Bernard HR. Data management and analysis methods. 2000

42. Saunders RR, Saunders MD, Donnelly JE, Smith BK, Sullivan DK, Guilford B, et al. Evaluation of an approach to weight loss in adults with intellectual or developmental disabilities. Intellectual and developmental disabilities. 2011; 49:103-12. [PubMed: 21446873]

43. Reichard A, Saunders MD, Saunders RR, Donnelly JE, Lauer E, Sullivan DK, et al. A comparison of two weight management programs for adults with mobility impairments. Disabil Health J. 2015; 8:61-9. [PubMed: 25242059]

44. Tanis ES, Palmer S, Wehmeyer M, Davies DK, Stock SE, Lobb K, et al. Self-report computerbased survey of technology use by people with intellectual and developmental disabilities. Intellectual and developmental disabilities. 2012; 50:53-68. [PubMed: 22316226]

45. Kagohara DM, van der Meer L, Ramdoss S, O'Reilly MF, Lancioni GE, Davis TN, et al. Using iPods $((\mathrm{R}))$ and $\mathrm{iPads}((\mathrm{R}))$ in teaching programs for individuals with developmental disabilities: a systematic review. Res Dev Disabil. 2013; 34:147-56. [PubMed: 22940168]

46. Wehmeyer ML, Smith SJ, Palmer SB. Technology Use by Students with Intellectual Disabilities: An Overview. Journal of Special Education Technology. 2004; 19:7-22.

47. Standen PJ, Brown DJ. Virtual reality in the rehabilitation of people with intellectual disabilities: review. Cyberpsychology \& behavior. 2005; 8:272-82. [PubMed: 15971976] 
Table 1

Intervention overview

\begin{tabular}{|c|c|c|c|}
\hline & \multicolumn{3}{|c|}{ INTERVENTION GROUPS } \\
\hline & $\mathrm{RD} / \mathrm{CD}$ & RD/eSLD & FTF/CD \\
\hline \multicolumn{4}{|l|}{ Behavioral sessions } \\
\hline Delivery method & FaceTime ${ }^{\mathrm{TM}}$ (iPad) & FaceTime $^{\mathrm{TM}}$ (iPad) & Home visits \\
\hline Delivery target & Adolescent+Parent & Adolescent+Parent & Adolescent+Parent \\
\hline Session duration & $30-45$ min. & $30-45 \mathrm{~min}$. & $30-45 \mathrm{~min}$. \\
\hline \multicolumn{4}{|l|}{ Session frequency } \\
\hline $0-6$ mos. & $2 \times$ per mo & $2 \times$ per mo & $2 \times$ per mo \\
\hline 7-12 mos. & $2 \times$ per mo. & $2 \times$ per mo. & $2 \times$ per mo. \\
\hline $13-18 \mathrm{mos}$ & $1 \times$ per mo & $1 \times$ per mo & $1 \times$ per mo \\
\hline \multicolumn{4}{|l|}{ Diet } \\
\hline Type & Conventional meal plan diet & $\begin{array}{l}\text { eSLD (portion controlled } \\
\text { entree's + low kcal shakes) }\end{array}$ & Conventional meal plan diet \\
\hline Energy intake (0-6 mos.) & $\begin{array}{l}500-700 \mathrm{kcal} / \mathrm{d} \text { below } \\
\text { estimated energy expenditure }\end{array}$ & $\begin{array}{l}500-700 \mathrm{kcal} / \mathrm{d} \text { below } \\
\text { estimated energy expenditure }\end{array}$ & $\begin{array}{l}500-700 \mathrm{kcal} / \mathrm{d} \text { below estimated } \\
\text { energy expenditure }\end{array}$ \\
\hline Energy intake (7-18 mos.) & $\begin{array}{l}\mathrm{kcal} / \mathrm{d} \text { to reflect estimated } \\
\text { energy expenditure } \times 1.4 \text { to } \\
\text { account for PA }\end{array}$ & $\begin{array}{l}\mathrm{kcal} / \mathrm{d} \text { to reflect estimated } \\
\text { energy expenditure } \times 1.4 \text { to } \\
\text { account for PA }\end{array}$ & $\begin{array}{l}\mathrm{kcal} / \mathrm{d} \text { to reflect estimated energy } \\
\text { expenditure } \times 1.4 \text { to account for PA }\end{array}$ \\
\hline Physical Activity & $300 \mathrm{~min} / \mathrm{wk}$ & $300 \mathrm{~min} / \mathrm{wk}$ & $300 \mathrm{~min} / \mathrm{wk}$ \\
\hline Self-monitoring (Diet) & Lose It! with iPad & Lose It! with iPad & Pencil/paper logs \\
\hline Self-monitoring (PA) & Fitbit with iPad & Fitbit with iPad & Pedometer-Pencil/paper logs \\
\hline Self-monitoring (Weight) & Wi-Fi enabled scale & Wi-Fi enabled scale & Portable scale \\
\hline
\end{tabular}

$\mathrm{RD}=$ remote delivery, $\mathrm{CD}=$ conventional diet, $\mathrm{eSLD}=$ enhanced Stop Light Diet, FTF = face-to-face, $\mathrm{PA}=$ physical activity 


\section{Table 2}

\section{Participant eligibility criteria}

\begin{tabular}{|c|c|}
\hline INCLUSION & \\
\hline Residence & Live with a parent in North East Kansas, i.e. $\leq 50$ miles from Kansas City, KS. \\
\hline Diagnosis & Mild to moderate IDD (IQ 40-74) as documented by a physician. \\
\hline Age & $13-18$ yrs. \\
\hline Weight & Overweight or obese (BMI $\geq 85^{\text {th }} \%$ ile) or waist circumference to height ratio $>0.50$ (central obesity). \\
\hline Ambulation & Must be able to walk. \\
\hline Communication & $\begin{array}{l}\text { The ability to communicate preferences (e.g., foods like or disliked, wants (e.g., more to eat, drink, and needs (e.g. } \\
\text { assistance with food preparation) through spoken or sign language. }\end{array}$ \\
\hline Heath status & Must provide physician clearance to participate. \\
\hline Internet access & Must have wireless internet access in the home. \\
\hline \multicolumn{2}{|l|}{ EXCLUSION } \\
\hline \multirow[t]{3}{*}{ Health status } & Insulin dependent diabetes, uncontrolled hypertension etc. \\
\hline & Diagnosis of Prader-Willi Syndrome. \\
\hline & $\begin{array}{l}\text { Eating disorder, serious food allergies, consuming special diets vegetarian, Atkins, etc.), aversion to common foods } \\
\text { (e.g., unwilling to consume dairy products, vegetables etc.). }\end{array}$ \\
\hline Recent weight loss & Participation in a weight management program involving diet and physical activity in the previous 6 months. \\
\hline
\end{tabular}


Table 3

Outcome assessment schedule

\begin{tabular}{|c|c|c|c|c|}
\hline & \multicolumn{4}{|c|}{ Study Month } \\
\hline & 0 & 6 & 12 & 18 \\
\hline \multicolumn{5}{|l|}{ Primary/Secondary outcomes } \\
\hline Weight/height/waist circumference & $\mathrm{x}$ & $\mathrm{x}$ & $\mathrm{x}$ & $\mathrm{x}$ \\
\hline \multicolumn{5}{|l|}{ Cost Analysis } \\
\hline \multicolumn{5}{|l|}{ Exploratory outcomes } \\
\hline Compliance-Diet/PA & $\mathrm{x}$ & $\mathrm{x}$ & $\mathrm{x}$ & $\mathrm{X}$ \\
\hline \multicolumn{5}{|l|}{ Behavioral session attendance } \\
\hline \multicolumn{5}{|l|}{ Self-Monitoring- Diet/PA } \\
\hline Parent Diet & $\mathrm{x}$ & $\mathrm{x}$ & & $\mathrm{x}$ \\
\hline Parent PA & $\mathrm{x}$ & $\mathrm{x}$ & & $\mathrm{x}$ \\
\hline Parent beliefs/attitude toward diet/PA & $\mathrm{x}$ & $\mathrm{x}$ & & $\mathrm{x}$ \\
\hline Medications & $\mathrm{x}$ & $\mathrm{x}$ & & $\mathrm{x}$ \\
\hline Structured interviews & & & & $\mathrm{x}$ \\
\hline
\end{tabular}

PA = Physical activity 